\title{
Walking Pattern Analysis Using an Acceleration Sensor Device
}

\author{
Ju-Hee Hong*, Kap-Soo Han** and Kyungho Kim ${ }^{\dagger}$
}

\begin{abstract}
In this paper, a device to analyze gait pattern was developed by using a 2-axis acceleration sensor attached to the foot. The 1st low-pass filter was adapted to limit the frequency band up to $5 \mathrm{~Hz}$. An algorithm to detect the peak value exceeding the threshold voltage of an X-axis acceleration sensor and a Z-axis acceleration sensor was developed and normal and abnormal walking patterns were thus differentiated. Also, MCU and Bluetooth were combined to transfer the data to other MCUs to display on an LCD; the size of the device could then be reduced. The new algorithm and the device allowed the individual walking patterns to be easily measured at a low cost and with less restriction on activities compared to conventional multiple pressure sensors or motion camera system.
\end{abstract}

Keywords: Accelerometer, Walking pattern

\section{Introduction}

Currently in society, the sedentary life style and modern diet have significantly changed the health of the human body and increased numerous adult diseases such as obesity, high blood pressure, angina pectoris, myocardial infarction, and diabetes [1]. Therefore, interest in sports such as field exercise, yoga, and fitness training is also increasing. Among these, walking is one of the most recommended exercises and many studies have been carried out regarding its physiological effects on the body [2].

The World Health Organization (WHO) suggested that 30 minutes of walking for 5 days per week could reduce the risk of heart attack by about $37 \%$. Leon et al. performed an experiment with 6 obese males $(\mathrm{BMI}=38)$ who were asked to walk for 90 minutes a day for 11 weeks. They reported that the exercise reduced body fat from $23.3 \%$ to $17.4 \%$ [3]. One of the major advantages of walking is that it is the most primitive type of human activity, and can be performed without the help of special devices, regardless of location and time [4].

Walking is defined as the movement of human limbs while performing biphasic forward propulsion of the center of gravity of the human body [5]. Walking patterns can be characterized by the differences in body movement patterns such as velocity, muscle and ground forces, and the surface of the ground [6]. The human walking pattern can be differentiated as either a natural or a specialized trained pattern and it can affect the spinal components such as vertebral body, ligaments, and muscles in the case of abnormal gait patterns $[7,8]$.

$\dagger$ Corresponding Author: Department of Electronics Engineering, Dankook University, Korea. (dkuhealth@dankook.ac.kr)

* Department of Electronics Engineering, Dankook University, Korea. (meanric@naver.com)

** Division of Biomedical Engineering, Chonbuk National University, Jeonju, Republic of Korea, Korea. (hanks@jbnu.ac.kr)

Received: December 26, 2015; Accepted: July 12, 2016
Therefore, many studies have been performed to analyze and suggest normal and correct walking patterns [10].

Generally, approaches using a motion camera system or pressure sensors are widely used in walking analysis. In the former, the image signal from the camera system is analyzed; however, this approach can be limited by the experimental situation and the duration time for the daily activities. With the approach of using multiple pressure sensors, the results are fairly dependant on the size and shape of the foot, while it is difficult to measure the walking pattern with 1-2 sensors.

Therefore, we aimed to develop and introduce acceleration sensors as a new device and propose a new algorithm to analyze the human walking pattern.

\section{Method}

Walking consists of the following four actions that make a pattern of movement with body weight: 1) producing the power to move forward; 2) keeping a vertical balance between the foot and the ground while making continuous postural changes; 3) minimizing the impact transferred from the ground to the lower limbs; 4) recovering the energy in the muscles to maintain the process of walking. Each action is fairly dependant on the walking pattern [10].

Fig. 1 shows the normal walking patterns based on the foot pressure distribution. The contact of the heel with the ground at the initial step and the loading response starts with the loading of the heel on the ground. The contact area is maximum in the mid-stance and the heel starts to lift up from the ground at this stance. As soon as the heel contact ends, the terminal stance begins.

\subsection{Walking pattern retrieval system}

In this study, an acceleration sensor was adopted to collect signals during walking activity. Noise must be 


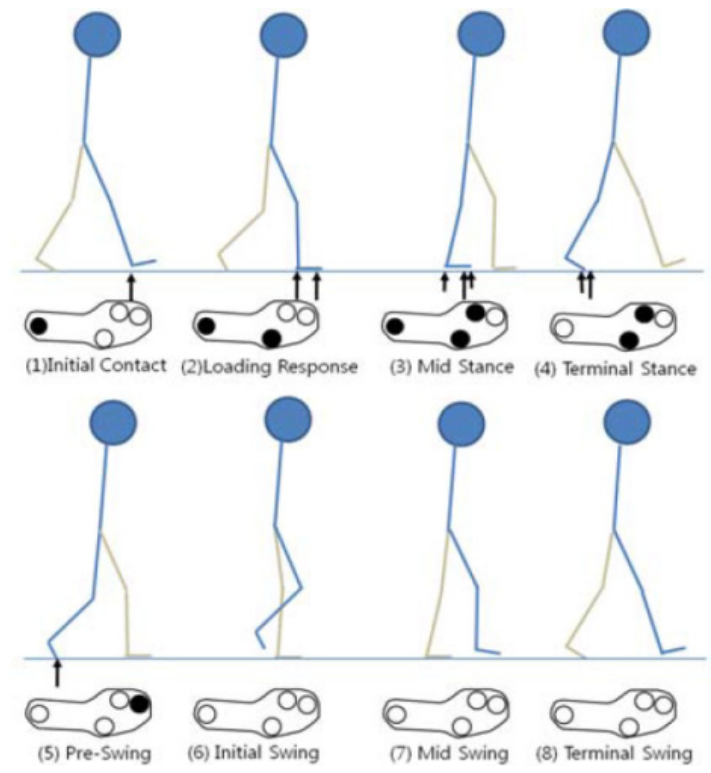

Fig. 1. The schematic of walking steps

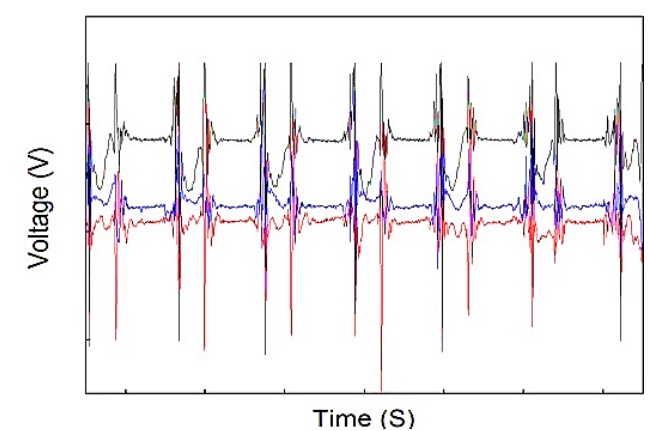

Fig. 2. Data before the low-pass filter. Red, blue, and black lines indicate the $\mathrm{X}, \mathrm{Y}$, and $\mathrm{Z}$ directions, respectively

considered in the $\mathrm{X}, \mathrm{Y}$, and $\mathrm{Z}$ signal directions due to the electrical and electromagnetic waves, and the line of electricity. Therefore, the signals need to be filtered using a 1st order active low-pass filter in the Operational Amplifier the band range close to $5 \mathrm{~Hz}$ was considered as the noise and removed.

In the case of the right leg, to which the acceleration sensors were attached, one to two cycles of gait were completed in 1-2 seconds; that is, one cycle of gait required 1.5 seconds. Therefore, the frequency band was restricted up to the maximum range of $5 \mathrm{~Hz}$ in a low-pass filter since the gait cycle ranged from 1 to $2 \mathrm{~Hz}$. The collected acceleration signals were sent to the microcontroller unit (MCU). The atmega128 (Atmel: http://www.atmel.com/) was used as the MCU for preprocessing for Blue-tooth communication.

\subsection{Analysis of gait pattern}

To analyze a gait cycle and pattern, a gait experiment
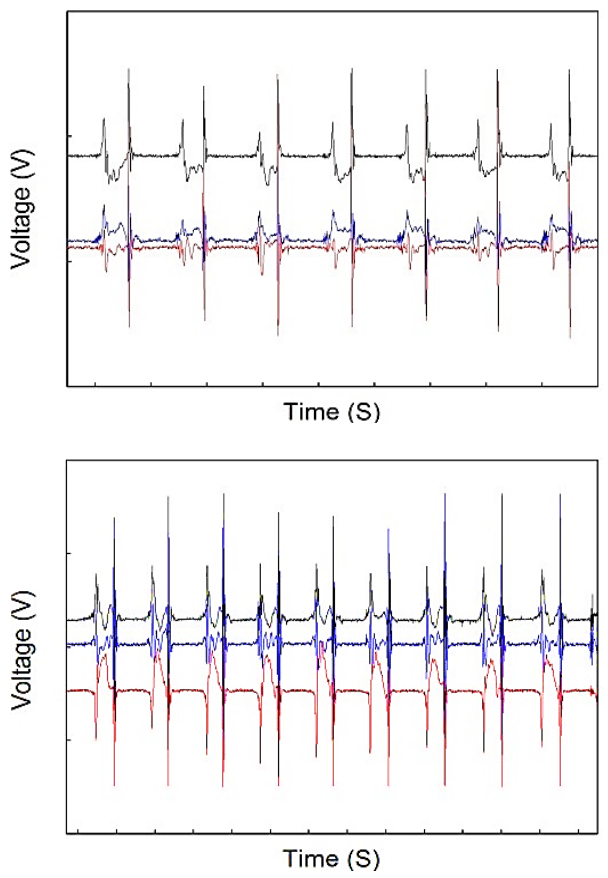

Figs. 3, 4. Data after the low-pass filter. Red, blue, and black lines indicate the $\mathrm{X}, \mathrm{Y}$, and $\mathrm{Z}$ directions, respectively

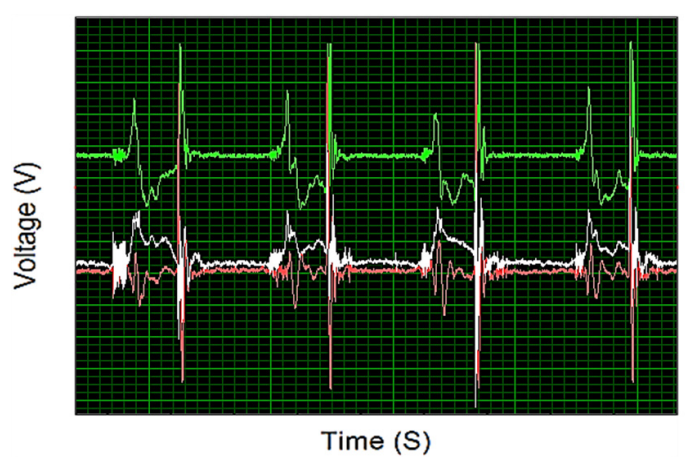

Fig. 5. Data confirmed by Labview Signal Processing

was conducted with 10 of healthy male and female subjects. Gait exercise was performed for 30 seconds and the data were analyzed using atmega 128.

The reference data were selected using the algorithm programmed by Labview signal processing.

Figs. 2, 3, and 4 show the data acquired from Labview signal processing using the Origin graph format. The $\mathrm{X}, \mathrm{Y}$, and $\mathrm{Z}$ directions of the acceleration data before the lowpass filtering are shown in Fig. 2 and those after the lowpass filtering are shown in Figs. 3 and 4.

Fig. 5 shows the data acquired using the Labview signal processing program; the red, white, and green lines indicated the $\mathrm{X}$-axis, Y-axis, and Z-axis.

The $X$-axis acceleration data of the right leg during normal gait is shown in Fig. 6 and those for the Z-axis are shown in Fig. 7. During the toe off phase, the toe of the forward right foot was in contact with the ground while the 
right heel is slightly lifted. The elevated wave is shown at the initial contact step and another elevated wave for the step of the left foot was recorded. A similar wave trend was observed for the right foot as shown in Figs. 6 and 7.

Only the $\mathrm{X}$-axis and $\mathrm{Z}$-axis of the signal were selected for the gait pattern analysis in this study. To set the reference voltage, the acceleration value was recorded and averaged while the subject was in the static state for 3 seconds at the sampling rate of $0.004 \mathrm{~s}$.

Fig. 8 shows the $\mathrm{X}$-axis of the acceleration data processed using Origin software; the reference voltage was set to be $+0.6 \mathrm{~V}$ as the threshold voltage. Fig. 9 shows the $\mathrm{Z}$-axis of the data and the reference voltage was set at $+0.9 \mathrm{~V}$ as the threshold voltage. The threshold voltages of $\mathrm{X}$-axis and $\mathrm{Z}$-axis is an algorithm created through the subject of this experiment.

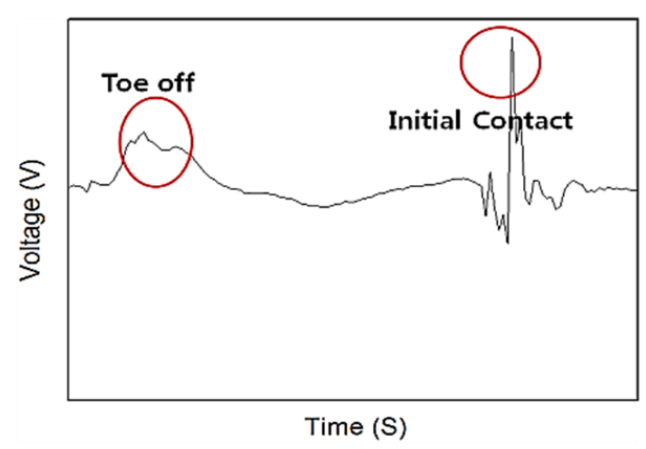

Fig. 6. X-axis correct gait pattern analysis.

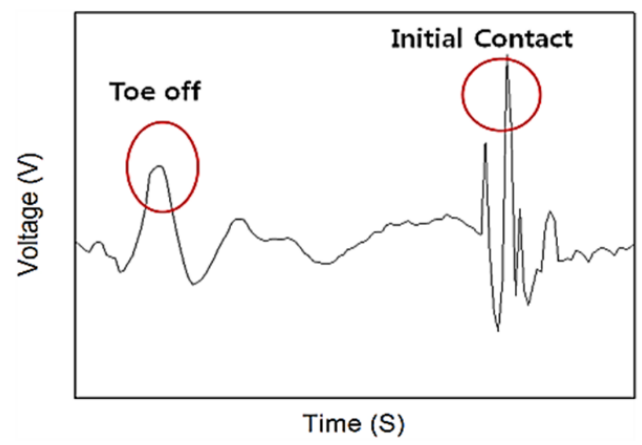

Fig. 7. Z-axis correct gait pattern analysis.

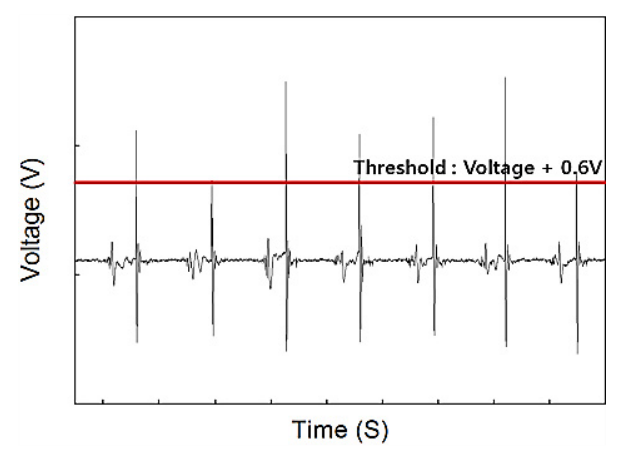

Fig. 8. $\mathrm{X}$-axis data. (1V div / $1 \mathrm{~S}$ div)
Figs. 10 and 11 show examples of the unstable gait, such as faltering steps and shuffle steps. The data was acquired by Labview signal processing and the Origin program. Results showed that the trend differed from that of the normal gait.

\subsection{Algorithm}

Fig. 12 showed the schematic of the proposed algorithm. Algorithm is counted the number, only $\mathrm{X}$-axis is above $0.6 \mathrm{~V}$ in one direction. The reference voltage was measured and set during the five seconds of static state. To acquire a sampling rate, the gait was performed for 10 seconds and the threshold for the $\mathrm{X}$-axis and $\mathrm{Z}$-axis was set as $+0.6 \mathrm{~V}$ and $+0.9 \mathrm{~V}$, respectively. The count $\mathrm{X}$ and count $\mathrm{Z}$ shown over the threshold of each axis were calculated. Once the

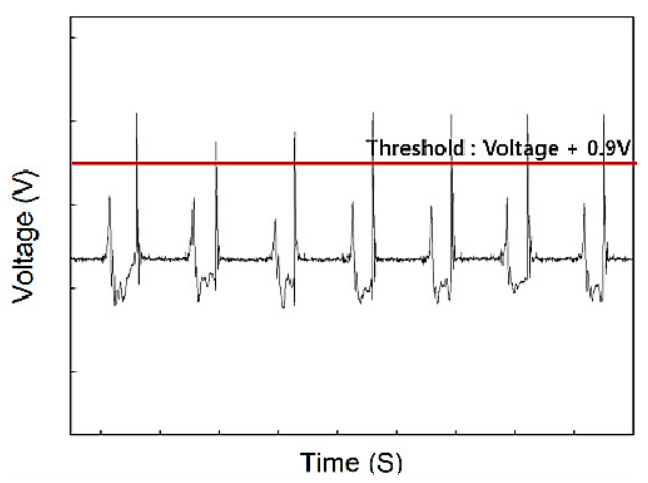

Fig. 9. Z-axis data. $(0.5 \mathrm{~V}$ div / $1 \mathrm{~S}$ div $)$

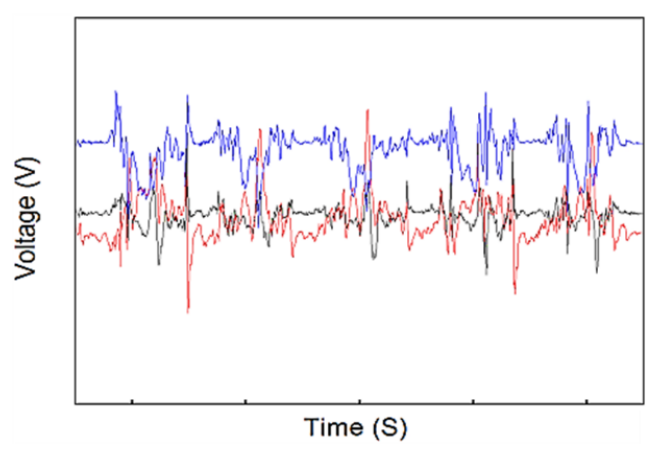

Fig. 10. Unstable gait data (Unstable gait).

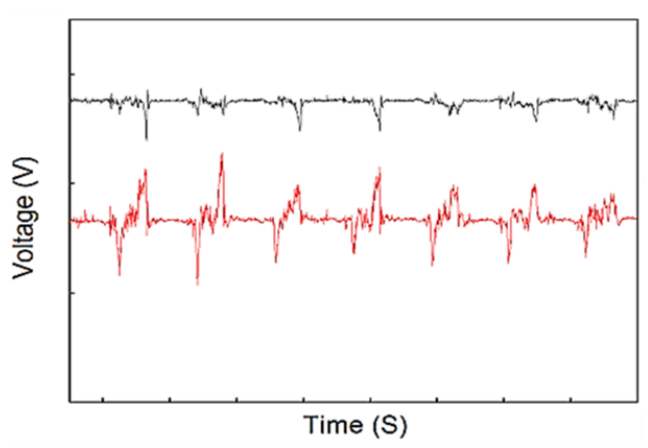

Fig. 11. Unstable gait data (shuffle) 


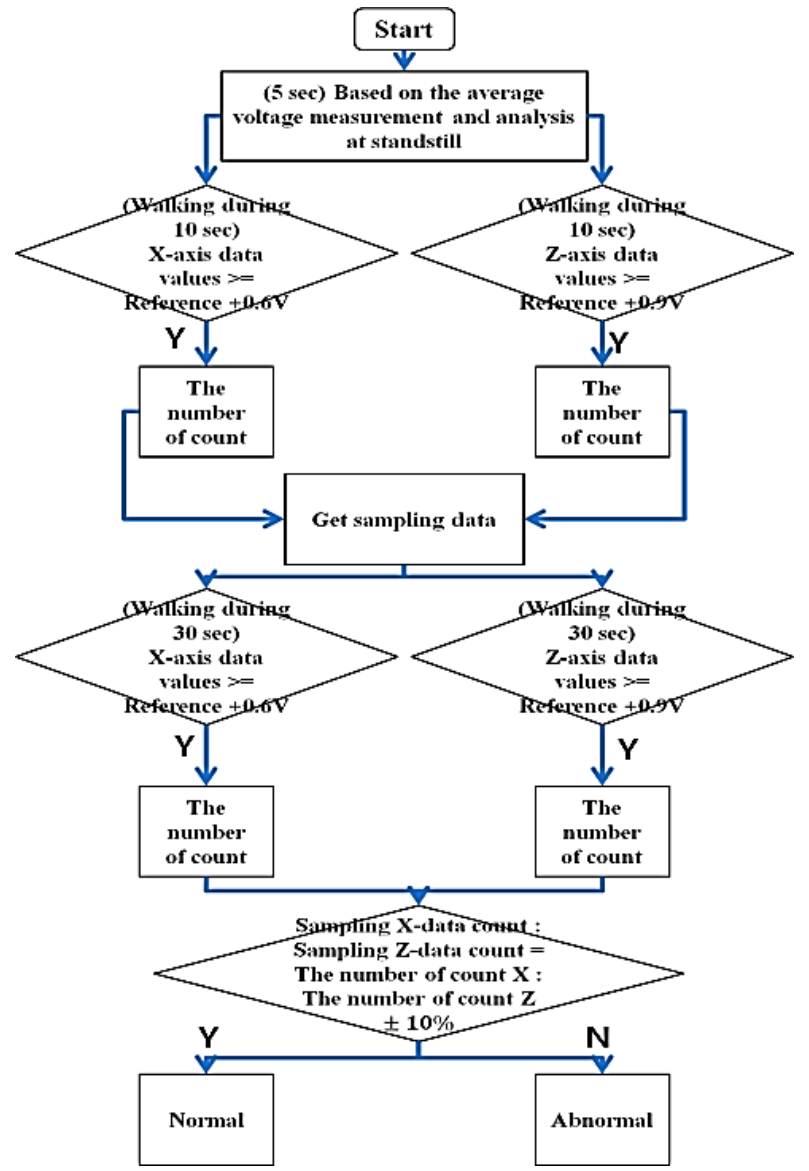

Fig. 12. Algorithm for walking pattern analysis

sampling rate was acquired, count $\mathrm{X}$ and count $\mathrm{Z}$ were divided by 10 and the number of peaks for one second was obtained. When the gait was started, the data was divided by 30 and the new calculated count $\mathrm{X}$ and count $\mathrm{Z}$ were obtained using formula (1). The proportion of count $X$ and sampling $X$-data count is the same as the proportion of count $\mathrm{Z}$ and sampling $\mathrm{Z}$-data count. At this time, the case of $Z$-count has a professional range 10 of error.

The normal and abnormal gaits were evaluated according to the rate of count $\mathrm{X}$ and count $\mathrm{Z}$ in the sampling data through formula (1) every 30 seconds. The peak point was detected above $2.9 \mathrm{~V}$ in the case where the reference voltage was more than $2.4 \mathrm{~V}$.

$$
\frac{\text { Count }-X}{\text { Sampling } X-\text { datacount }}=\frac{\text { count }-Z \pm 10 \%}{\text { Sampling } Z-\text { datacount }}
$$

Fig. 13 shows the analyzed data to confirm the results shown in Fig. 12 using Labview signal processing and $\mathrm{C}$ language. Figs. 14 and 15 show the waves of the $\mathrm{X}$ and $\mathrm{Y}$ axes using Labview signal processing for 30 seconds of normal gait. In this gait analysis, two steps were not detected and two peak points were detected in the $\mathrm{X}$-axis; these were considered as detection errors.

Table 1 shows a comparison of the $\mathrm{X}$-axis and $\mathrm{Z}$-axis

\begin{tabular}{|c|c|c|c|c|}
\hline \multicolumn{4}{|c|}{ data - 메모장 } & 口|⿴囗口 \\
\hline 파일 & 편집( $(\mathrm{E})$ & 서식(O) 보기(V) & 도움말(H) & \\
\hline $\begin{array}{l}\text { 번호 } \\
0 \\
1 \\
2 \\
3 \\
4 \\
5\end{array}$ & $\begin{array}{l}\text { t ime } \\
1.096 \\
2.44 \\
3.764 \\
5.084 \\
6.412 \\
7.7 n \pi\end{array}$ & 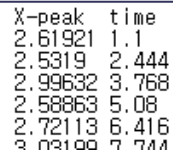 & $\begin{array}{ll}\text { Y-peak } & \text { ti me } \\
2.10867 & 1.1 \\
1.90491 & 2.448 \\
2.24111 & 3.776 \\
2.0781 & 5.092 \\
2.00679 & 6.42 \\
1.09120 & 7.712\end{array}$ & $\begin{array}{l}\text { Z-peak } \\
3.0400 \mathrm{E} \\
2.9024 \mathrm{E} \\
2.9534 \mathrm{c} \\
3.0451 \mathrm{E} \\
3.0349 \mathrm{E} \\
3 \text {. } 240 \mathrm{E}\end{array}$ \\
\hline
\end{tabular}

Fig. 13. Data detected by the $\mathrm{C}$ language.

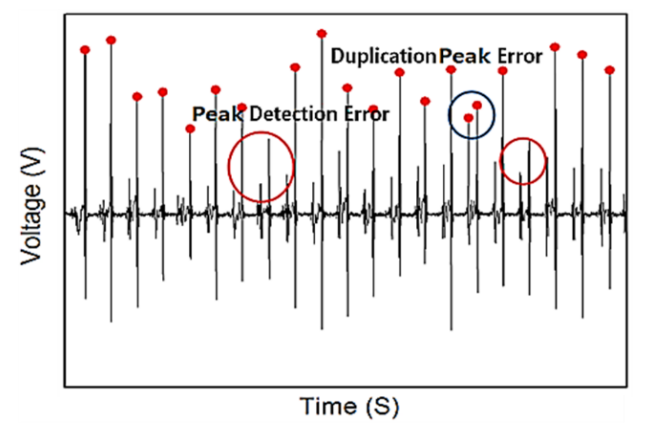

Fig. 14. X-axis peak detection results

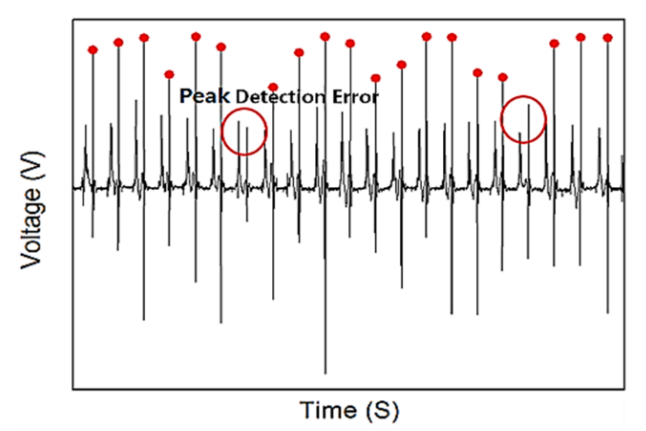

Fig. 15. Z-axis peak detection results.

Table 1. Representative maximum muscle activities among major

\begin{tabular}{c|c|c|c|c|c|c|c|c|c|c}
\hline Person & 1 & 2 & 3 & 4 & 5 & 6 & 7 & 8 & 9 & 10 \\
\hline X-axis & 23 & 20 & 21 & 20 & 16 & 21 & 22 & 21 & 20 & 24 \\
\hline Z-axis & 22 & 19 & 19 & 19 & 19 & 23 & 21 & 21 & 21 & 24 \\
\hline
\end{tabular}

acceleration peak points during a normal gait for 30 seconds obtained from Labview signal processing and the proposed algorithm.

When the data from the Labview signal processing and the posture analysis presented in the algorithms was compared, a $90 \%$ accuracy was obtained. When the gait was slow, the distortion and the noise increased. It appeared that the $10 \%$ error induced the noise due to the swing down of the foot and the inconsistent swing down. Also, the detected value at the toe-off was higher than that of the reference value or the value for the initial contact was lower than the peak value. Therefore, the algorithm was developed to resolve the case where the peak value of the $\mathrm{X}$-axis and $\mathrm{Z}$-axis at the toe-off phase was higher than the reference peak value. 


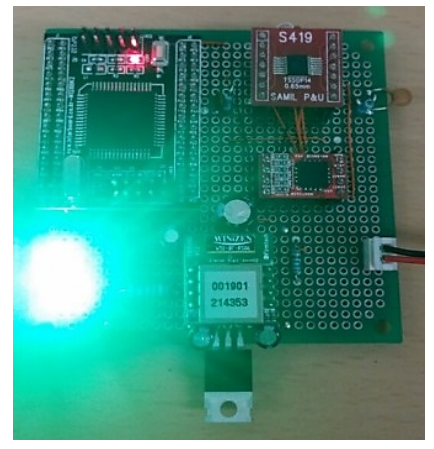

Fig. 16. The schematic of developed device

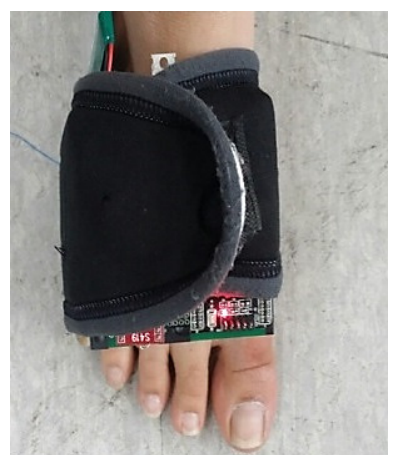

Fig. 17. The schematic of the device attached on the foot

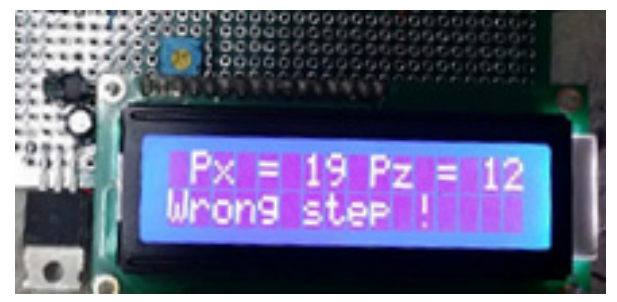

Fig. 18. Bluetooth communication

\subsection{Experiments and data analysis}

Gait analysis was performed with 10 subjects with acceleration sensors attached to their foot. As shown in Fig. 1, a normal gait was attempted and the gait pattern was analyzed using the MCU through the Bluetooth communication.

Fig. 16 shows the developed device and the schematic of the attached device on the foot. The atmega128 was operated at $5 \mathrm{~V}$ and $\mathrm{FB} 155 \mathrm{BC}$ was operated at $3.3 \mathrm{~V}$ and $\operatorname{lm} 1117$. The $\operatorname{lm} 7805$ regulator was used to communicate am-3axis, the 3 -axis acceleration sensors. Fig. 17 shows a schematic of the device attached to the foot used to measure the data. To facilitate the measurement of the foot, the support band was used to hold the device on the foot and the rear side of the device was wrapped with insulating tape and sponge to prevent line breakage.

Fig. 18 shows the acquired sampling data of the $\mathrm{X}$-axis and Z-axis at the peak point for 30 seconds while walking. The LCD panel shows a schematic of the normal and abnormal gait at the peak point. $\mathrm{Px}$ and $\mathrm{Pz}$ indicate the $\mathrm{X}$ axis and $\mathrm{Z}$-axis of the acceleration at the peak point, respectively.

\section{Conclusion}

Abnormal walking is known to contribute to disorders of the spine and other joints. Therefore, it is important to maintain normal and correct postures and walking patterns during daily activities. In this paper, the acceleration data of the $\mathrm{X}$-axis and Z-axis were collected during walking gait. The collected data from the newly developed device showed more accurate individual gait pattern trends with low cost and less motion limitation compared with other previous devices. Moreover, the precise circuit was able to be structured using a low path filter and an algorithm to analyze the wave of 30 seconds of walking using an MCU; the device was developed to communicate between the MCU and LCD.

The reference voltage was identified and the sampling data was set for 30 seconds of individual gait pattern, and the gait pattern was then analyzed. In this paper, when the data from the gait and the posture analysis presented in algorithms was compared, a 90\% accuracy was obtained.

The result from this study can be used as a standard to evaluate abnormal walking patterns. Also, due to their easy facilitation, the components and device developed in this study can be integrated into a wearable device for a health diagnosis system.

For future study, electromyography (EMG) sensors will be adopted to measure the EMG signal in the lower extremity during walking and the algorithm will the developed to also analyze gait pattern.

\section{Acknowledgements}

The present research was conducted by the research fund of Dankook University in 2014.

\section{References}

[1] Ran Choi "An analysis of walking styles and ground reaction force on Obese students in elementary school", Chinju National University of Education, 2008.

[2] Hyeok-Jeong Gwon, "Impacts of Walking Exercise on obesity", Seonam University, 2009.

[3] A S Leon, J Conrad, D B Hunninghake, R Serfass "Effect of a Vigorous Walking Program on Body Composition, and Carbohydrate and Lipid Mdtabolism of Obese Young Man", Aam J clin Nutr, 32, 176-178, 1979.

[4] Hye-Mi Kim, "The Effect of Postural Walking 
Exercise on Back Pain and Recovery of Trunk Balance among Patientes with Low Back Pain", SoGang University, 2011.

[5] Hang-Jeok Choi, "A Study on Gait Analysis System using 3-axis Accelerometer", Inha University, 2015.

[6] Chun-Seong Lee, "Idiopathic Scoliosis", Journal of Korean Society of Spine Surgery, Vol. 6(2), 1999.

[7] Mi-jung Park, Jeong-Suk Park, "Effect of a Posture Training Program on Cobb Angle and Knowledge of Posture of Elementary School Students", Journal of Korean Academy of Nursing, Vol. 33(5), 2003.

[8] Gwang-Chul Shin, "Effects of 10 weeks of combined remedy exercise on angle of truncal rotation, body balance, electromyography, and physical fitness in Elementary students with scoliosis", Kyung-Hee University, 2014.

[9] Jong-Hee Han, "A study on the gait analysis for patients with movement disorders using 3-axis accelerometer system", Seoul National University, 2005

[10] Murray MP, Kory Rc, Clarkson BH, and Sepic SB, "Walking Patterns of Normal Men", vol. 46, no. A, pp. 335-360, 1964.

[11] Geun-Seop Heo, "The study on Sensor System for Human Gait Analysis", Kyungpook National University, 2011

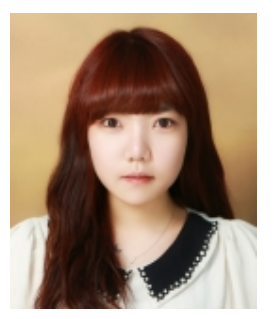

Ju-Hee Hong received M.S. degrees in electronic engineering from Dankook University at 2016. Her research interests Walking pattern analysis field.

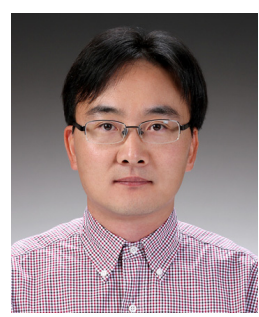

Kap-Soo Han received his B.S. and M.S. degree from Chonbuk National University and Ph.D. from the University of Iowa. His research interests include medical devices, biomechanics, rehabilitation, multi-body dynamics and musculoskeletal modeling.

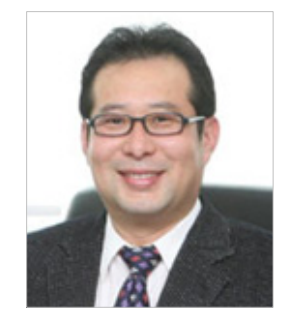

Kyungho Kim received M.S. and Ph. D. degrees in biomedical engineering from Keio University, Japan. He worked at SAIT from 2000 to 2006 . He is currently an professor in Electronics Engineering at Dankook University. His research interests ubiquitous health, signal processing, sensor system and 